\title{
CHANGES IN BLOOD COAGULATION FOLLOWING CORONARY THROMBOSIS MEASURED BY THE HEPARIN RETARDED CLOTTING TEST (WAUGH AND RUDDICK TEST) ${ }^{1,2}$
}

\author{
By JOSEPH H. OGURA, NORMA R. FETTER, M. A. BLANKENHORN, AND \\ HELEN I. GLUECK
}

(From the Department of Internal Medicine, University of Cincinnati College of Medicine and Cincinnati General Hospital)

(Received for publication November 1, 1945)

Recently many authors have studied the phenomenon of accelerated coagulability of the blood in a variety of conditions. It is now well known that numerous factors may play a rôle in increasing the coagulability of the blood. This tendency toward an increasing coagulability of the blood will be referred to as "acceleration" throughout this paper. Among factors which may accelerate the coagulation of blood should be mentioned stasis of blood, trauma to vessel walls, dehydration, anemia, and the release of excessive quantities of thromboplastin in the blood such as occur after trauma, surgery, infection, or childbirth. Each of these factors may act independently or in conjunction with the others. De Takats (1) demonstrated a change in the clotting mechanism in a variety of thrombotic states such as venous thrombosis, cerebral thrombosis, arterial embolus and coronary thrombosis. Brambel et al. (2) demonstrated a tendency toward acceleration of coagulation using Quick's prothrombin test with 12.5 per cent diluted plasma in crush injuries associated with gangrene, diabetic and arteriosclerotic gangrene and frost bite. Shapiro $(3,4)$ has also demonstrated a tendency to accelerated coagulability of the blood using diluted plasma, and thought it significant in thromboembolization. He found in 5 cases of embolization a lowered prothrombin time. Hirschboeck (5) found in 9 out of 10 cases of pulmonary embolism a decrease in clot retraction time. Shafiroff et al. (6) found, using the Lee-White technique, that blood from a limb affected with thrombosis or phlebitis, as compared with blood taken from a normal limb in the same subject, showed accelerated coagulation.

\footnotetext{
1 Aided by a grant from the William S. Merrell Company in Cincinnati.

2 A brief abstract of this paper was read by title at the Proceedings of the Central Society for Clinical Research at Chicago, 1945.
}

Doles (7), however, found in 13 cases of coronary thrombosis that 12 to 48 hours after the episode, the prothrombin times in percentage of normal were reduced. Hines and Kessler (8) found in 8 patients with evidence of either coronary or cerebral thrombosis, a tendency to increased coagulation as measured by Quick's prothrombin determination and the De Takats heparin tolerance method (1). They found this in association with a high erythrocyte count. Nay and Barnes (9) have recently reviewed a series of 100 cases of coronary thrombosis, in which complications of a thrombotic or embolic nature occurred in 37 cases.

Heparin has been suggested as a therapeutic measure by Best (10). Experimentally, Solandt and Best (11) prevented coronary thrombosis in previously heparinized dogs. Knowing that anticoagulants are being used in the management of coronary thrombosis as well as other diseases, it seems desirable to know, if possible, whether such patients do indeed have accelerated clotting and to what degree. Furthermore, if such an acceleration were demonstrated, it would be desirable to know the duration of such a change.

Our present study was motivated by an autopsy study by one of us (12) of 100 hospital cases of coronary thrombosis. Extension of the coronary clot in a proximal direction was found in 12 per cent of the cases. In 18 per cent a new thrombosis occurred, probably while the patient was in the hospital. Mural thrombosis on the wall of the ventricle occurred in 60 per cent of the cases, often associated with auricular mural thrombosis. In an additional 5 per cent, atrial mural thrombosis occurred without ventricular mural thrombosis. This gives a total of 65 per cent for all mural thrombosis. Peripheral thrombo-embolic manifestations occurred in 45 per cent of the cases, which came to autopsy because of the consequences of coronary thrombosis. With this in mind, serial 
study of blood coagulation was made on coronary thrombosis cases from hospital entry until discharge (or death) to see if some significant alteration did occur.

\section{METHODS OF OBSERVATIONS}

Sixteen hospital patients were selected as controls, and 27 different cases of coronary thrombosis were followed serially in our study. The Waugh-Ruddick test $(13,14)$ was used throughout in order to determine if any alteration of blood coagulation occurred in coronary thrombosis. The studies were made at room temperatures ranging from 70 to $75^{\circ} \mathrm{F}$., average $72^{\circ}$ (15). Lee-White and capillary clotting times were run simultaneously with the Waugh-Ruddick test. We at first familiarized ourselves with the method on 7 recent convalescents, who had completely recovered from their illness, and were ambulatory most of the day. Our results were in essential agreement with the authors. Frequent sedimentation rates and electrocardiograms were taken. In the earlier part of our study, the standard methods for determining prothrombin time and platelet counts were done in a few cases, but were discontinued because our resources did not permit. Nearly all studies were carried out in the late fall, winter, and early spring.

In our controls were 7 "normal" people who were placed in bed at absolute bed rest on hospital entry for varying conditions, i.e., 3 for psychoneurosis, 1 each for Little's disease, acute gastritis, thoracic aneurysm, and diaphragmatic hernia. Patients with major infections, surgical operations, and hemorrhage were purposely excluded, since Waugh and Ruddick have shown accelerated coagulation in a series of such cases (14). All had normal cardiac findings. Seven other cases had hypertension and arteriosclerotic heart disease with congestive failure, all on digitalis. Two other cases had severe angina pectoris, with 1 on digitalis. The 9 "cardiacs" were on absolute bed rest from the time of admission until observations were completed. Previous to hospital entry, the "normal" group had been ambulatory most of the day, and the cardiac control group was so at least part of the day before hospitalization. The patients with coronary thrombosis had also been ambulatory until the onset of acute symptoms. Patients taking digitalis were included in our control observations because 6 cases of coronary thrombosis were also taking digitalis.

All 27 cases of coronary thrombosis were diagnosed by the usual clinical and electrocardiographic evidence, and were treated by accepted therapeutic measures. Coagulation studies, using the Waugh-Ruddick technique (13), were made as early as possible from the date of entry, and were continued until discharge or death of the patient. At first the observations were made every day. Later, when it became apparent that this was unnecessary, tests were run 2 to 3 times weekly for 4 weeks, then weekly until discharge. More frequent observations were made during the 2nd and 3rd week and when complications ensued. In several instances, control observations were made months after discharge. In these few instances there was little difference in the coagulation studies on discharge after recovery, from those taken on the same patient months later. Ten of the 27 cases were observed at the Jewish Hospital.8

In all but 2 cases the exact date of onset of occlusion could be fixed on clinical and electrocardiographic evidences. The error in these 2 cases is a matter of 1 to 2 days. In several cases, the first observations were not made until 4 or 5 days after the thrombotic episode, usually because the patient had not entered the hospital. The majority of cases were observed within the first week of the initial attack. All questionable cases of coronary occlusion were excluded from our series. One case after discharge, on resuming her duties, developed another attack and was counted as a new patient.

\section{METHOD OF PLOTTING AND EVALUATION}

In the Waugh-Ruddick test (13) in order to recognize the phenomenon of accelerated coagulability of blood, controlled deceleration of the process with heparin in "slow motion" magnifies finer changes which can be more accurately measured. In brief, this test consists of recording the coagulation time of the blood in a series of 9 tubes, to which increasing quantities of heparin are added, A curve is then constructed, using the coagulation time in minutes on the ordinate, and the tubes with varying concentration of heparin on the abscissa. When it became apparent to us that there were distinct differences in the clotting curves following a thrombotic attack (Figure 1), the statistical significance was next considered. For this purpose the coagulation end-point of Tube 9 containing $7 / 10$ unit of heparin was selected, because here the "slow motion" is the slowest, and hence can be read with the least error as we practiced the technique of Waugh and Ruddick. Figure 1 illustrates a typical series of curves obtained during the course of the disease in 1 patient. The smaller the figures in minutes (i.e. Tube 9) the greater the tendency toward acceleration of the clotting tendency. This is spoken of as "flattening of the curve" as acceleration develops. Tube 9 is used in making Figures 2 to 4 , and in evaluating results. However, it is evident that the other tubes bear a systematic relationship to Tube 9 in terms of coagulation times. Tube 5 or 6 might well be chosen, but the differences are not as great as in Tube 9 where there is a greater concentration of heparin.

Realizing that certain difficulties may be experienced in obtaining samples of blood, since trauma incident to venous puncture may increase the thromboplastin in a particular sample to be examined, certain precautions were observed as suggested by the authors of the method. A large gauge (No. 18) needle was used, the blood being expelled through the needle. At least $15 \mathrm{ml}$. of blood

${ }^{3}$ We wish to thank Drs. H. Weiss, M. Salzer, L. Schiff, D. Goldman, F. Donath, P. Jaeger of the medical staff of the Jewish Hospital for consenting to this investigative procedure. 
PATIENT: G.C.
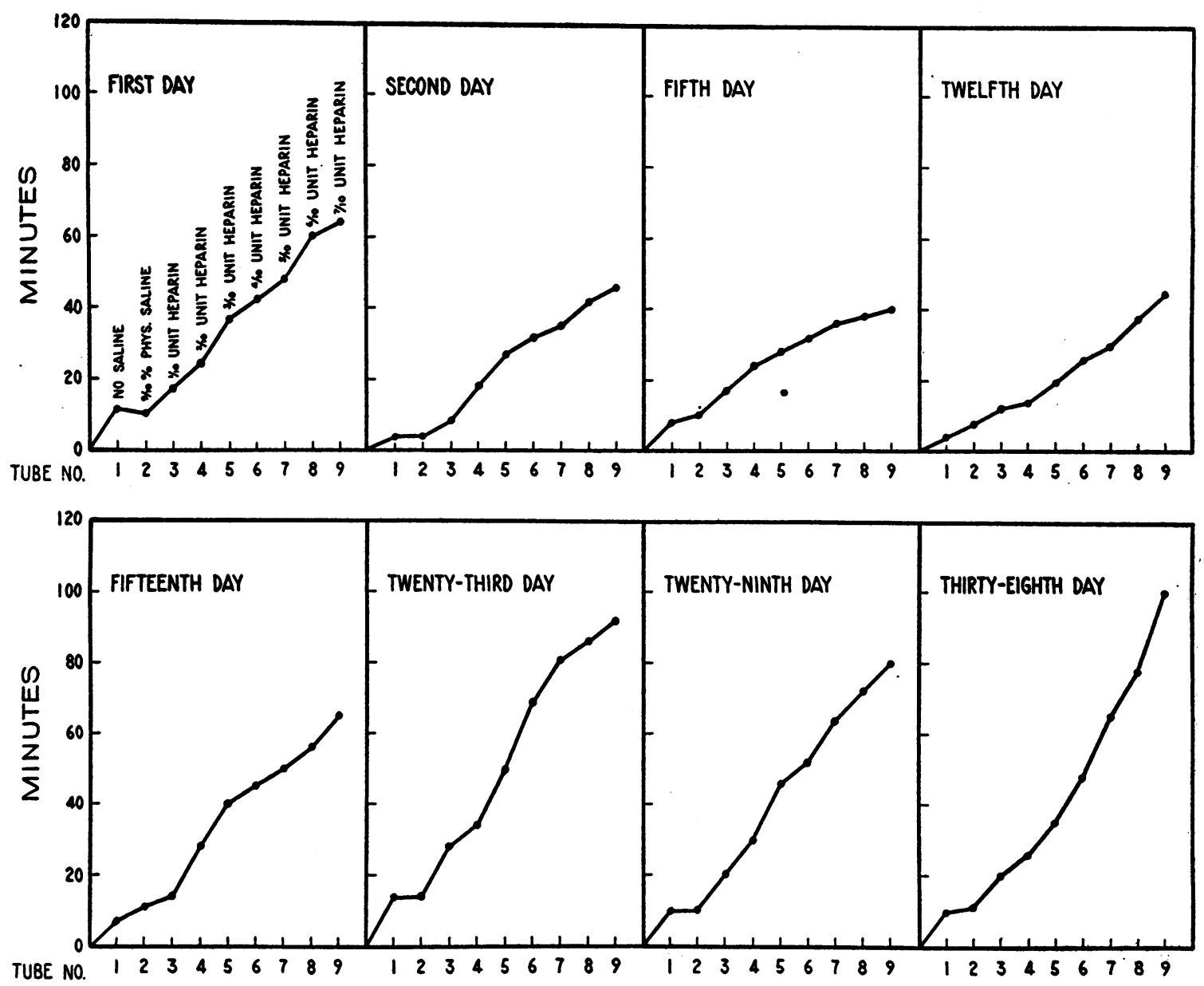

Fig. 1. Typical Changes in Blood Coagulation in a Case of Coronary Thrombosis

were utilized for each test. The blood was not used for the determinations if less than this amount of blood was obtained. We were particularly careful to prevent foaming of the blood since this altered the results. The glassware used was new, and especially prepared as suggested by Waugh and Ruddick. All samples of blood were drawn and tested by two of the authors of the present paper in order to insure uniformity of observation.

\section{The controls}

In our control group the 7 "normals" and 9 "cardiacs" differed only as to degrees of elevation initially and in the 3rd day in bed (see Figure 2). The general shape of the control curves are similar except that a more precipitous drop occurred with the cardiacs over the 3rd day to the 9th day. Nearly all of our controls showed mild flattening with acceleration of the curves from the 3rd day on. Four cases taking digitalis dipped into the zone we arbitrarily called acceleration of coagulation, such as occurred so commonly when there was coronary thrombosis. We do not know the significance of this, nor do we see any particular effect of digitalis therapy in the patients with coronary thrombosis who were also taking digitalis.

\section{Coronary thrombosis}

Out of the total of 27 cases, 21 (77.8 per cent) showed the phenomenon of accelerated coagulability during some point early in the hospital stay, and usually before the 12th day after the occlusion in an individual case. Very commonly, acceleration occurred by the 3rd day, and remained accelerated from several days to weeks. On a 
composite graph (Figure 2) the greatest dip turned to normal by the end of the 3rd week. One seemed to occur on the 13 th day. In one case, acceleration remained as late as the 32 nd day. The majority of cases, with the exception of 4 , recase, who showed acceleration for a longer period than the others, after returning to normal, developed another attack on his 37 th day after his

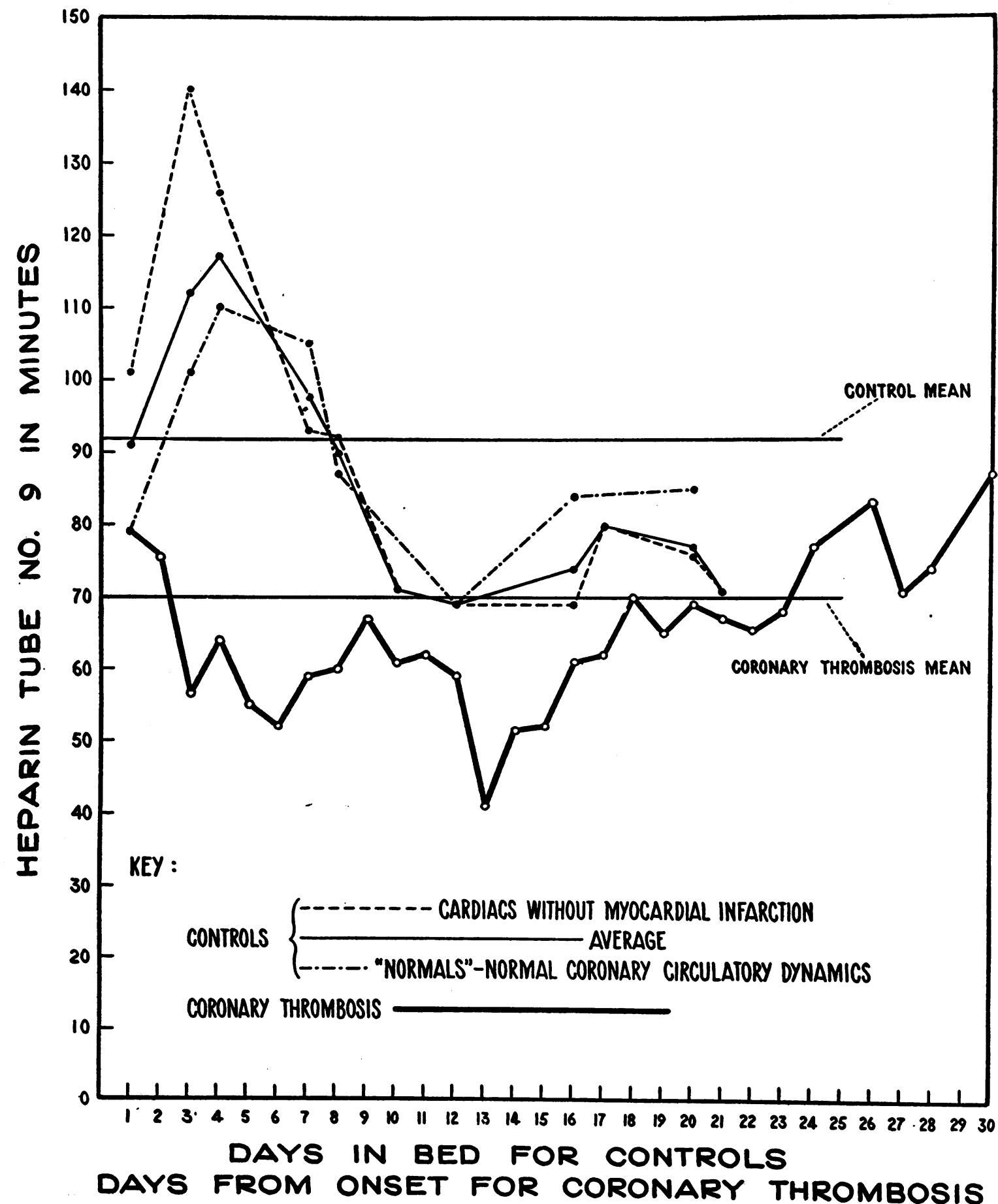

Fig. 2. Changes of Blood Coagulation in Controls and Coronary Thrombosis 


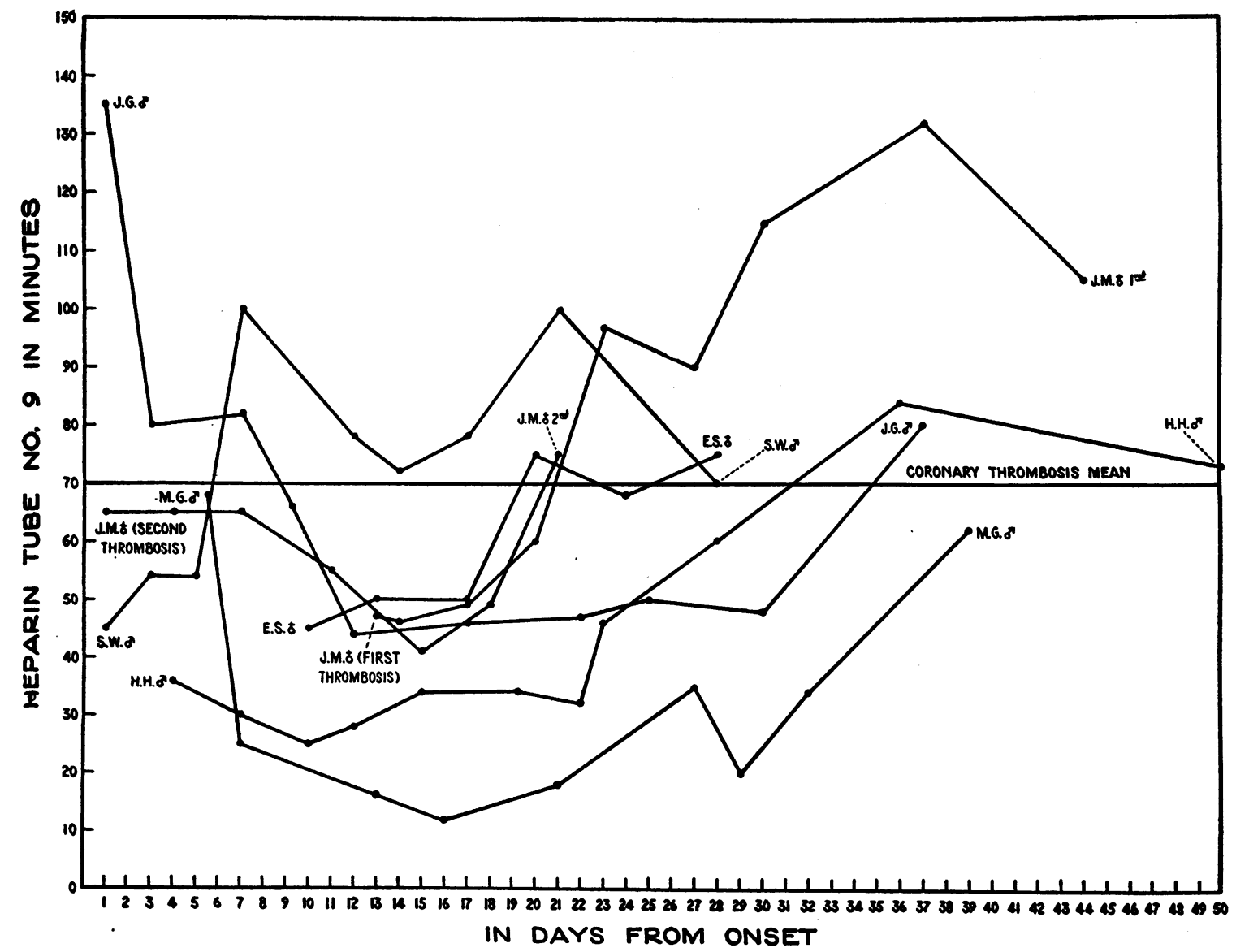

Fig. 3. Changes in Blood Coagulation in Several Uncomplicated Coronary Thrombosis Cases

first episode and again showed acceleration (Figure 4, D.B., male). Two were accelerated for a period of a few days beyond the 3rd week. The last case did not show acceleration until the 12th day after the attack, and consequently returned to normal at a later date than usual.

In order than one may visualize changes that occur in an individual case (uncomplicated coronary thrombosis), the values for Tube 9 have been plotted in serial fashion for several patients (Figure 3).

The nature of coronary heart disease did not make it seem feasible to test all the patients every day. On the other hand, we do not think the curve would be materially changed by daily testing. The points on the curves for coronary thrombosis in Figure 2 are the average values of Tube 9 for each day after onset in all of the 27 cases. The number of patients tested on each day varies from 8 to 14 , the greatest number being read from the 7 th to the 20th day. Our data appeared valid when analyzed statistically. ${ }^{4}$ The data were analyzed

\section{- Statistical analysis.}

If our results are analyzed statistically, we find the following :

Mean control ................92.2 mean minutes Mean coronary thrombosis ........70.3 mean minutes Standard deviation control .........24.58 minutes

Coronary .................25.16 minutes

Probable error control ............ plus 2.34 minutes Coronary ................. plus 1.16 minutes

Coefficient of variation control .......26.64

Coronary .................... 35.9

Probable error difference ..........2.67 minutes

The difference between the coronary group and the control group with reference to mean time for coagulation of Tube 9 on the heparin scale is 21.9 minutes. The probable error of this difference is plus 2.67 minutes. Using the threshold of 4 times the probable error for statistical significance, this difference is seen to be markedly significant, since the difference is 8 times its own probable 
by Dr. T. J. Le Blanc, ${ }^{5}$ Professor of Preventive Medicine, University of Cincinnati, College of Medicine.

\section{OTHER CLOTTING METHODS}

In the individual experiments, the Lee-White and the capillary clotting times run simultaneously with the Waugh-Ruddick test failed to reveal any strikingly significant change when the latter test was accelerated or normal. From our controls (Figure 2) one is struck by the majority of cases being above 60 minutes in Tube 9 of the WaughRuddick test.

To compare the Lee-White and the capillary tube methods with Waugh-Ruddick method, we chose 60 minutes (for Tube 9) as the dividing line above which we considered normal and prolonged, and below which we considered it accelerated. When our Lee-White and capillary results were divided according to the above selection and our findings compared, the following was revealed:

Waugh-Ruddick normal or

$$
\text { L. anb W. Cap. }
$$

prolonged .............9.45 minutes 4.05 minutes Waugh-Ruddick accelerated..8.75 minutes 3.35 minutes Difference ........... 0.7 minute 0.7 minute

While this difference may not seem significant, it is important that the average time was actually

error, meaning that the odds against a deviation as great or greater than this are about 14 million to one.

As to variability (the "two universes"), at first glance it might appear that the coronary cases show much greater variability than the controls. While the variability in this group is greater, it is not as much as would appear on inspection. The two standard deviations are relatively close together, and when these are expressed as coefficients of variability the respective values are 26.64 for the controls and 35.9 for the coronary group. In other words, the high variability inherent in the control group does not have much significance, and it is doubtful that any clinical interpretation may be made of such a difference.

It is recognized that the respective variables are high (standard deviations), but in spite of these high levels which enter into the respective probable errors of the means, the probable error of the difference between the two means is either sufficiently low, or the difference sufficiently high, to make this difference strongly implied. (See R. Pearl, "Introduction to Medical Biometry and Statistics," Ed. 2, 1930, P. 283, W. B. Saunders Co.)

- Dr. T. J. Le Blanc, Professor of Preventive Medicine, University of Cincinnati, College of Medicine, kindly aided us in the statistical analysis. lower for both the Lee-White and capillary clotting times when acceleration was present in the Waugh-Ruddick test. It should be stated, however, that the final interpretation of the results was based on the Waugh-Ruddick test, rather than any other method.

A few platelet counts and a few standard prothrombin times were done, but we did not employ this method enough to determine if accelerated clotting could be demonstrated.

An attempt was made to correlate trends of acceleration of coagulation with the erythrocyte sedimentation rate, but no clear cut relation could be established. Generally, acceleration returned to normal before the sedimentation rate did. Within the first 48 hours of the attack when an increase of the sedimentation rate was present, acceleration may be present. Later, when acceleration usually occurred, the sedimentation rate was almost always increased. After the 3rd or 4th week, when there was no demonstrable acceleration, the sedimentation rate often was still increased.

\section{RELATION OF COMPLICATIONS TO ACCELERATION}

Figure 4 shows a random selection of cases who developed complications of coronary thrombosis while being observed.

Patient, J. P., male, had a recent anterior myocardial infarct on admission. Progressive tendency to acceleration of coagulation developed. On the 5th day after the initial episode, the patient developed another clinical attack, and the electrocardiogram revealed evidences of a posterior infarction. The curve approached normal on the 21st day, and no further complications were noticed.

Patient, R. B., male, had a recent posterior myocardial infarction on admission. A sharp tendency to acceleration developed. On the 6 th day after the onset another attack occurred, and the following day the electrocardiogram revealed an anterior infarction.

Patient, R. B., female, showed a recent anterior myocardial infarction on admission. A flat curve was present when on the 12th day after onset, patient developed a pulmonary embolus and the following day a shift to R.A.D. in the electrocardiogram, with clinical evidences of pulmonary infarction. The curves thereafter returned to normal on the 29th day. A few days after discharge, the patient developed another new coronary thrombosis.

Patient, J. G., male, revealed an anterior infarct on entry. Progressive flattening developed. When maximum acceleration was present, patient developed more evidences of posterior wall involvement. 


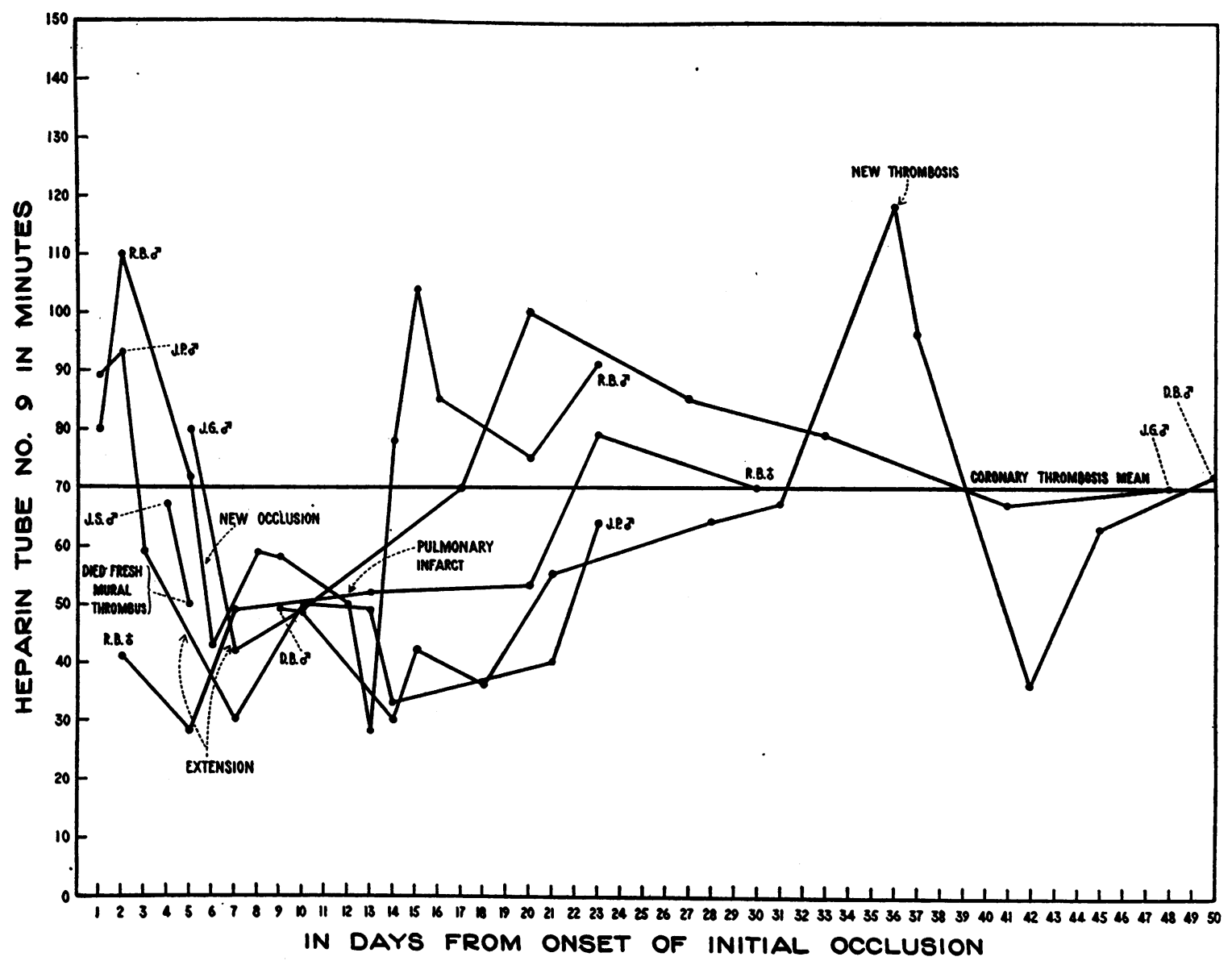

Fig. 4. Changes in Blood Coagulation in Complicated Cases of Coronary Thrombosis

Patient, J. S., male, presented an interesting picture. A history of dull precordial pain 3 days before hospital admission was obtained. Patient also had diabetes mellitus. Because of left-sided failure, the patient was placed on digitalis. Electrocardiograms revealed an anterior infarction. The coronary occlusion only contributed to the patient's exitus, inasmuch as it was a small apical infarct (no coronary thrombosis, but moderately severe coronary atherosclerosis of the left anterior descending vessel was present). Of major importance was the fact that the patient died of uremia due to Kimmelstiel-Wilson's disease. Acute fibrinous pericarditis was also present. This patient was included in this group since a large very fresh mural thrombosis of only several days' duration was found.

Patient, D. B., male, who previous to admission had an old anterior infarction, developed a new posterior infarct responsible for his admission. Acceleration observed immediately on admission was maintained for 31 days before returning to normal. On the 36th day after the onset of the posterior infarct, another attack developed with new electrocardiographic findings (extension) with progressive tendency towards acceleration. Seven days later maximum flattening occurred.
The first 4 cases would suggest that when the phenomenon of acceleration was present, the patient was liable-to a new thrombosis or to extension or to thrombo-embolization. The curve of patient D. B., male, suggests that after returning to normal, the initiating mechanism of the new occlusion or thrombosis is not necessarily dependent on the coagulatory mechanism. However, the phenomenon of acceleration following occlusion is again demonstrated.

Five cases of coronary thrombosis died while in the hospital. Two died of acute heart failure, 3 died of coronary thrombosis or massive pulmonary embolus. All except 1 showed acceleration at some time. Two days before death, curves were normal in 3 . One showed acceleration continuously until death. One died in the 4th day of onset without showing acceleration. Two cases came to autopsy while under study, and one only showed mural thrombus. 
DIGITALIS, XANTHINES, AND CORONARY THROMBOSIS

It has recently been claimed that digitalis ( 16 to 19 ) and the xanthine (20) drugs favor accelerated coagulation, and therefore due regard was given to that point in our study. It would appear from our control group who required it, that as the patients were digitalized some acceleration . develops.

Of the 6 patients with coronary thrombosis taking digitalis (Figure 5), 4 were on maintenance doses when they developed their occlusion, and 2 were digitalized on hospital entry after occlusion.

Of the 6,2 cases (J. S. and N. P.) revealed acceleration curves that differed from our usual type in neither degree of acceleration nor duration. Both cases died, however.

Three of the remaining 4 on maintenance doses of digitalis returned to normal after the usual ac- celeration, showing no continued acceleration even though digitalis continued to be taken.

The remaining case (K. P.) was not observed until late in the 2 nd week. No acceleration was found during her entire stay.

Again, these cases are too few from which to draw any conclusions. One may say that the accelerating response following coronary occlusion would seem to follow its usual course, despite digitalis. If digitalis has an additional accelerating effect, more cases and further studies are necessary to demonstrate the degree of such addition.

Four of the patients with coronary thrombosis received adequate doses of the xanthines during their hospitalization for a period of 3 or more days. None showed any more acceleration, as measured by the Waugh-Ruddick method, than would be expected from coronary thrombosis alone.

Three patients from the cardiac control group

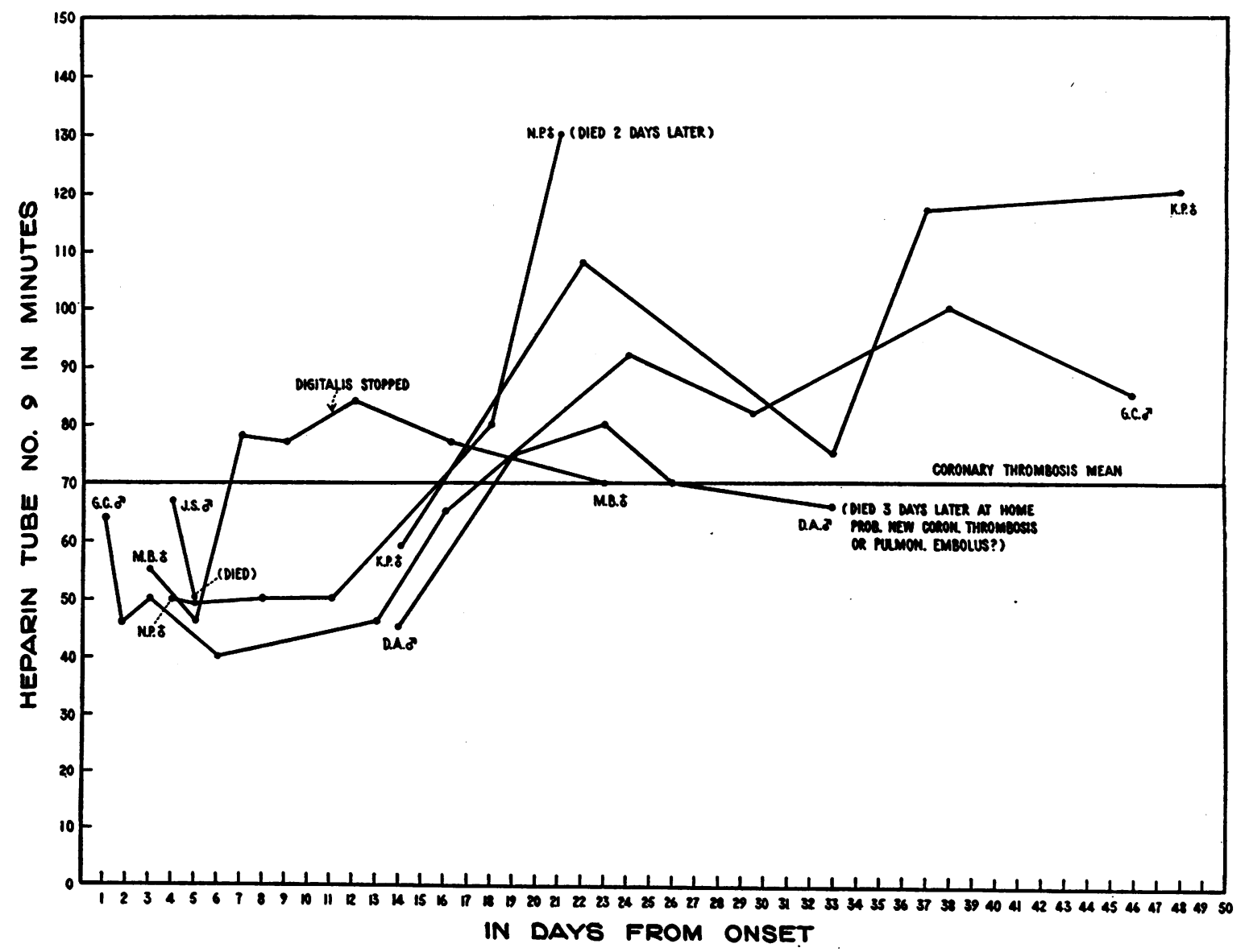

Fig. 5. Changes in Blood Coagulation in Coronary Thrombosis Cases on Digitalis 
were also given large doses of xanthines: 2 received aminophylline intravenously, and 1 received theobromine in large doses. No acceleration was noted, other than what was expected from bed rest alone.

No conclusions can be drawn from such a small series of cases, although Field et al. (20) have demonstrated hyperprothombinemia in animals receiving xanthine drugs. We do not know if any parallel can be drawn between their methods and the Waugh-Ruddick technique.

\section{DISCUSSION}

It is admitted that the Waugh-Ruddick test is empirical, artificial and may not represent what occurs in vivo; the same may be said for other coagulation tests. When we compare the LeeWhite and capillary tube methods with the Waugh-Ruddick tests, we find that both of the former tests reveal a faster clotting time by 0.7 minute when acceleration by the Waugh-Ruddick method was present. This difference does not look very significant. However, when both tests were on the average faster, this assumes more significance than if only one were faster. Whether it is this small difference we are measuring on the Waugh-Ruddick test remains to be seen.

In reality, the -Waugh-Ruddick test is an in vitro De Takats Heparin Tolerance Test. The "hyporeactors" which De Takats (1) found in various thrombotic states would be consistent with our findings.

Since we are employing a new method in studying the coagulation of the blood, a discussion of the method seems in order. It is true that by the addition of heparin, small differences are magnified. However, the differences are also evident in Tubes 3 or 4 , where smaller concentrations of heparin are used. Unfortunately, the various methods which have been devised for measuring the clotting time, while satisfactory for demonstrating a prolongation of the process, are difficult to interpret when acceleration occurs. This is probably due to the fact that, under normal conditions, the coagulation time is relatively short, and unless conditions are very carefully controlled, acceleration of coagulation may be very difficult to detect.

As we have gained experience with the method we have noticed a remarkable consistency in the determinations from day to day. This is especially evident in the ambulatory patients. In the control group, bed rest alone causes some acceleration of the curve (Figure 2). After this initial acceleration, which reaches its maximum about the 9th day, the curves remain more or less stabilized. In the coronary group, as the curves later approach normal, they are quite similiar to the curves taken on the first day of the episode. All their observations suggest that chance alone does not determine the results. Our findings in the control group on rest alone, and in the ambulatory patients, are in accord with those of the authors of the method (14). In considering the significance of the method, we are probably measuring the concentration of thromboplastic substances in the blood. Brinkhous, Smith, Warren, and Seegers (21) have demonstrated that heparin along with its serum complement is antagonistic to thromboplastin.

Waugh and Ruddick (14) believe that the flattening of the curve is due to an increase in thromboplastin. They have shown that by increasing the concentration of thromboplastin, a flattening of the curve results. Likewise, removal of platelets from the blood by centrifuging, markedly prolongs the coagulation of the blood in their test. If we consider that thromboplastin could easily come from infarcted damaged myocardium, or from disintegrating platelets which initiate mural thrombus formation, this explanation becomes even more attractive. Thromboplastin, although present in the platelets, can be derived from many tissues, and hence, is essentially a tissue extract. It is probable that the increased thromboplastin present in the blood following coronary thrombosis and myocardial injury is in no way different from that which occurs following hemorrhage, muscle injury, trauma, or surgical or obstetrical intervention. An added fact which supports this thesis is that marked acceleration of coagulation in the cases of coronary thrombosis is not observed immediately after the accident, but 2 to 3 days later, after myocardial tissue damage has resulted from the thrombosis, and the mural thrombus begins to form. Hence, accelerated coagulation probably plays no rôle in the causation of the thrombosis, but is rather the result of the thrombosis and its subsequent tissue damage. However, 
once acceleration is present, it may possibly lead to further complications.

Until some method of isolating, or more positively, demonstrating, thromboplastic alteration of the blood is developed, further comment would only be superfluous. Whether the acceleration of "prothrombin time" reported by Shapiro (4) in the 5 cases of thrombo-embolization would occur in coronary thrombosis will require further study. Doles (7), however, found in 13 cases of coronary thrombosis, using Smith's whole blood method for prothrombin determination, that after an occlusion the prothrombin time was reduced to an average of about 57 per cent of normal 12 to 48 hours after the attack. Consequently, he used vitamin $\mathrm{K}$ in treating his cases. Quick (22) has several serious objections to the Smith Bedside test. Although Doles' (7) studies were on coronary thrombosis, his findings would seem to vary from others who have found accelerated "prothrombin times." It should again be emphasized that the acceleration of coagulation found in coronary thrombosis is not specific, since it is found in thrombosis and embolus elsewhere, in postoperative patients following hemorrhage, acute infection, or childbirth.

Our groups of 27 hospital observations on coronary thrombosis is by no means a large series upon which to draw any definite conclusions. The findings suggest that following a thrombotic or occlusive episode a tendency to acceleration develops. An increasing tendency towards acceleration occurs reaching an initial low level by the 3rd day, and remaining relatively low, to reach an apparent maximum dip by the 14th day. A general maintenance of acceleration seems evident until the 17th day. Thereafter, the curve becomes indistinguishable from the control group. The observations on the first day of these 27 patients and the control group seem similar. It may be said that the blood coagulative mechanism shows little alteration from normal the first day after the attack, and from the 17 th day onward, by this method. This would suggest that the clotting mechanism preceding the attack, and after the 3rd week does not differ from the normal. The occurrence of 5 cases with definitely proven complications, such as a new thrombus in a period in which there is acceleration, suggests that acci- dents tend to occur when favored by accelerated coagulability of the blood. Clinicians are aware of this dangerous period in recovering from coronary thrombosis.

If future investigations should confirm these findings, the implications are obvious. The dangers of anticoagulant therapy are well known. The liability to retrograde progression of coronary thrombus, and to formation of a new thrombus also, is speculative, but the incidence of mural thrombus and embolization is so high in our experience that some new approach to the problem of therapy is desirable. From unpublished data (12), formation of mural thrombi seemed more frequent 48 hours after the onset of the thrombosis, fewer between 24 and 48 hours after onset, and rarely within the first 24 hours. This would coincide with the more frequent embolization occurring between the 7 th and the 10th day of onset. The chances of preventing these complications would seem better the earlier the therapy is commenced.

\section{CONCLUSIONS}

Twenty-seven cases of acute coronary thrombosis were studied in hospital to find evidence of accelerated blood coagulation. In 77.8 per cent of the cases, an acceleration as measured by the Waugh-Ruddick test occurred usually by the 2nd or 3rd day, lasting to about the 17th day. Occasionally, acceleration was delayed until the 12th day of the illness, and persisted only for several days. Acceleration was prolonged beyond the 3 rd week in a few cases.

Of 16 controls, 9 being in various stages of heart failure, none showed acceleration to the degree seen in coronary thrombosis. The statistical significance of this finding is set forth. An explanation is not attempted.

Such complications as new coronary thrombosis or extension of the original thrombus or pulmonary infarction, while admittedly too few for definite conclusions, developed in 5 patients when acceleration was present, suggesting that this factor may be contributory.

Our findings further suggest that the first attack is not initiated by accelerated coagulability of the blood in the average case, and that after the 4th week the clotting mechanism is indistinguishable from normal. 
We are deeply indebted to Drs. M. Logan and M. J. Boyd of the Department of Biochemistry and Dr. Virgil Hauenstein and J. Harold Kotte, cardiologists at the Cincinnati General Hospital.

\section{BIBLIOGRAPHY}

1. De Takats, G., Heparin tolerance; test of clotting mechanism. Surg., Gynec., and Obst., 1943, 77, 31.

2. Brambel, C. E., and Loker, F. F., Application of dicoumarin in trauma and gangrene. Arch. Surg., 1944, 48, 1.

3. Shapiro, S., Sherwin, B., Redish, M., Campbell, H. A., Prothrombin estimation; procedure and clinical interpretations. Proc. Soc. Exper. Biol. and Med., 1942, 50, 85.

4. Shapiro, S., Hyperprothrombinemia, premonitory sign of thromboembolization (description of method). Exper. Med. and Surg., 1944, 2, 103.

5. Hirschboeck, J. S., and Coffey, W. L., Jr., Clot retraction time in thrombophlebitis and pulmonary embolism. Am. J. M. Sc., 1943, 205, 727.

6. Shafiroff, B. G. P., Doubilet, H., Barcham, I. S., and Co Tui, Coagulability of venous blood of normal and diseased legs; study on 191 subjects. Ann. Surg., 1943, 118, 482.

7. Doles, H. M., Prothrombin determinations in acute coronary occlusions. South. M. J., 1943, 36, 709.

8. Hines, L. E., and Kessler, D. L., Venesection for plethoric patients. Arch. Int. Med., 1945, 75, 248.

9. Nay, R. M., and Barnes, A. R., Incidence of embolic or thrombotic processes during the immediate convalescence from acute myocardial infarction. Am. Heart J., 1945, 30, 65.

10. Best, C. H., Heparin and thrombosis. Brit. M. J., 1938, $2,977$.

11. Solandt, D. Y., and Best, C. H., Heparin and coronary thrombosis in experimental animals. Lancet, 1938, 2, 130.
12. Ogura, J. H., Unpublished data.

13. Waugh, T. R., and Ruddick, D. W., A test for increased coagulability of the blood. Canad. M. A. J., 1944, 50, 547.

14. Waugh, T. R., and Ruddick, D. W., Studies on the increased coagulability of the blood. Canad. M. A. J., 1944, 51, 11.

15. Whittaker, J., The effect of temperature on the Waugh and Ruddick test for increased coagulability of blood. Canad. M. A. J., 1945, 52, 185.

16. De Takats, G., Trump, R. A., and Gilbert, N. C., The effect of digitalis on the clotting mechanism. J. A. M. A., 1944, 125, 840.

17. Macht, D. I., Experimental studies on heparin and its influence on toxicity of digitaloids, congo red, cobra venom, and other drugs. Ann. Int. Med., 1943, 18, 772.

18. Werch, S. C., Reduction of the coagulation time of rabbit's blood by digitalis. Quart. Bull., Northwestern Univ. M. School, 1943, 17, 50.

19. Massie, E., Stillerman, H. S., Wright, C. S., and Minnich, V., Effect of administration of digitalis on coagulability of human blood. Arch. Int. Med., 1944, 74, 172.

20. Field, J. B., Larsen, E. G., Spero, L., and Link, K. P., Studies on sweet clover disease; hyperprothrombinemia induced by methylxanthines and its effect on the action of 3, 3'-methylenebis-(4-hydroxycoumarin). J. Biol. Chem., 1944, 156, 725.

21. Brinkhous, K. M., Smith, H. P., Warner, E. D., and Seegers, W. H., Inhibition of blood clotting: unidentified substance which acts in conjunction with heparin to prevent conversion of prothrombin to thrombin. Am. J. Physiol., 1939, 125, 683.

22. Quick, A. J., The Hemorrhagic Diseases and the Physiology of Hemostasis. C. C. Thomas, Springfield, Ill., 1942. 